\title{
Optimization of UAV structure and evaluation of vibrational and fatigue characteristics through simulation studies
}

\author{
Ashraf Mahmud Rayed ${ }^{1}$ (D), Balasubramanian Esakki ${ }^{2,}{ }^{*}$ (D), Arunkumar Ponnambalam ${ }^{3}$ (D), Sajal Chandra Banik ${ }^{1}$, \\ and Karim $\mathrm{Aly}^{4}$ (D) \\ ${ }^{1}$ Department of Mechanical Engineering, Chittagong University of Engineering and Technology, Chittagong, Bangladesh \\ 2 Department of Mechanical Engineering, Vel Tech Rangarajan Dr. Sagunthala R\&D Institute of Science and Technology, \\ Chennai, Tamil Nadu, India \\ ${ }^{3}$ Department of Mechanical Engineering, KPR Institute of Engineering and Technology, Coimbatore, Tamil Nadu, India \\ 4 Alexandria University, Alexandria Governorate, Egypt
}

Received: 28 May 2021 / Accepted: 12 August 2021

\begin{abstract}
Unmanned Aerial Vehicles (UAV) is generic air vehicles that are significantly developed for military and civil purposes. In recent times, advancements in the field of UAVs are exceptional and tremendous. Nevertheless, numerous researches have been performed mainly to reduce the weight of the UAV structure. The flight time and allowable payload rely on the UAV structure's weight, which is considered a significant factor. Hence, in this paper, the UAV model's static structural behavior is cultivated utilizing the morals of Finite Element Analysis (FEA) to determine the total deformation and Von-mises stress. Three different polymer materials, namely Poly Lactic Acid (PLA), Acrylonitrile Butadiene Styrene (ABS), and Polyamide (PA), are chosen for FEA analysis of 3D printed UAV structure. The thrust generated from the motors varies from $2 \mathrm{~kg}$ to $5 \mathrm{~kg}$, and an evaluation of structural strength characteristics is performed. The FEA study has shown that maximum deformation and maximum stress are obtained at the propeller casings and the basement of the structure, respectively. After comparing the details on deformation and stress from all three materials, the research also reveals that PLA is the best material for conception. Furthermore, topological optimization is performed on the UAV structure to reduce mass and minimize stress without compromising mechanical strength. The vibrational and fatigue characteristics of optimized UAV structure is examined. The unified body of the UAV frame will reduce the assembly time and make manufacturing much more effortless.
\end{abstract}

Keywords: Topology optimization / additive manufacturing / FEA / modal analysis / fatigue analysis and simulation

\section{Introduction}

Unmanned Aerial Vehicle (UAV) is now being widely used in multifaceted environments and applications. Because of its ability to operate in hazardous locations and successful operations in extreme conditions, various industries and defense sectors use UAVs for surveillance and reconnaissance operations. Intelligent UAVs are really the next important hit in Artificial Intelligence, aiming to generate new possibilities in a variety of applications, particularly in engineering structures, by lowering risks and costs. The well over $\$ 45$ billion valuation of UAV utilization is likely to be dominated by structural design [1]. The emergence of the Internet of Things (IoT) and, in particular, its rapid development and access to useful and, simultaneously time,

\footnotetext{
* e-mail: esak.bala@gmail.com
}

affordable precision agriculture technology such as airborne crop monitoring and intelligent screw driving operations [2] are gaining interest. Park et al. intend to classify UAV operations in mining by three segments: exploration, exploitation and rehabilitation of mining processes. They observed that UAVs are utilized for remotely sensed data, aviation geophysical surveys, rock slope analyses, work fulfillment analysis, subsurface measuring and environmental agreements, water, watershed management and coastal erosion at mining operations for detailed geological assessment [3]. Valsan et al. offers a standalone drone customized solutions to cope with earthquakes, landslides, floods, etc. This technology supports the rescue procedure by tracking the regions impacted and returning a signal to the evacuation unit when any casualties have been identified [4]. Sigala et al. apply the Delphi approach and rely on specialists in the field of business, commerce and academics. Future 
autonomous UAS missions will have great impact in defense and disaster services [5]. Esakki et al. [6] developed a unitary Quadrotor Chassis design, which minimizes assembly time. The mechanical strength of different grooved frames is analyzed with a view to achieve a high strength-to-weight ratio. Balasubramanian et al. introduced a technique for developing lightweight UAV structures that synergistically combines design iterations with fused deposition modeling. This analysis demonstrated a manufacturing procedure based on $3 \mathrm{D}$ printers and inexpensive Polylactic acid FDM filament material (PLA) [7]. As quadcopter UAV flight time and allowable payload relies on its weight, the UAV structure's weight has to be minimized. The reduction of mass of the UAV can be accomplished in two different ways. Mass can be decreased by using light-in-weight electronics instruments, or it can be reduced by using a frame that has been designed for low mass. The frame of a UAV is one of the main load-pressurebearing parts [8] and it constitutes up to $73 \%$ weight of the design. In order to reduce the structural weight of the UAV frame, optimization studies are necessary. Topology optimization is an appropriate technique for enhancing shape and limiting the mass of the design of UAVs. It is a crucial structure method, and it has gained quick ground in the most recent decades. Design space and non-design space needs to be defined before performing optimization [9]. It is unique in relation to measuring advancement and Figure improvement. As it is a freestyle material dissemination course of action, topology optimization empowers the development and amalgamation of the inside solids and voids all through the structure's advancement. Therefore, a lot greater and progressively complex structure space can be found, and better physical execution can be anticipated, and other essential advancements [10-12]. As the model is the extended structure space, the increased topological plan has been objected for specific reasons, which happen during the production and when the related CAD model is post altered. It is trying to guarantee that a topologically improved model can be made and satisfactory for mechanical use. As often as possible, analysts need to make a noteworthy stride now and then where the natural shape is characterized into basic geometries and remade that plan from common CAD natives. Regularly the sizable optimality is hard to track down in this stage. A vital examination is performed on manufacturability-arranged topology advancement to solve these concerns; both the thickness-based and level set systems [13-15] and interconnected examinations are carried out $[16,17]$. Coordinating unsurprising machining the length scale issue, no-undercut limitation, and highlight driven plan various topology optimization studies are performed in the literature [18-23]. After arriving at an optimized structure, an effective method of realizing the part swiftly is by means of a disruptive technology in the present scenario is additive manufacturing. AM technique is recommended for fabrication of the UAV structure in comparison to subtractive manufacturing and the later has more difficulties in terms of tooling and additional fixtures $[24,25]$. Presently, AM is the best method for the creation of any complex shape UAV parts in short span [26]. Ahmed et al. [27] developed a small-scale UAV model using 3D printing technology for wind tunnel testing. They have realized that $3 \mathrm{D}$ printed UAV is more cost-effective as it consumes less material and fabrication costs. Different types of UAVs and even sophisticated type UAVs are printed with 3D printing technology, as it proved its improvement in aerodynamics performance and structural efficiencies. Ahn et al. [28] examined Fused Deposition Modeling (FDM) process parameters and performed a Design of Experiment (DOE) to optimize the tensile and compressive strength of Acrylonitrile Butadiene Styrene (ABS) parts. They found that the air gap and raster orientation mostly influences tensile strength. The ABS material exhibits anisotropic property, and hence they predicted that the strength of the part depends on raster direction. Chockalingam et al. [29] worked on the enhancement of the anisotropic properties of ABS parts. Experiments are designed for four parameters using Central Composite Design (CCD) and optimized using genetic algorithm. Yang designed flapping wing micro aerial vehicle [30] of $20 \mathrm{~cm}$ wingspan and its mechanism module was fabricated using injection moulding technique. Hsiao et al. established an architectural technique for achieving self-directed flying wing MAVs that weigh less than $10 \mathrm{~g}$. They also proposed a balanced hypothesis for maintaining the fluttering MAV's vertical movement [31]. Yang et al. fabricated the micro mechanisms using injection molding technique which is costly [32] and time consuming.

In the present study, a static structural analysis is carried out on the 3D printed UAV structure using FEA tools of ANSYS software. The total deformation and vonmises stress are evaluated for three distinct polymers to arrive at a polymer that has high strength UAV structure. This study further extended to perform topological optimization of UAV structure as the primary concern is to reduce mass. Finally, a unified body of UAV structure is identified, which has the least possible mass that can balance all the loads, pressure, and thrust adequately. The vibrational and fatigue characteristics of the optimized UAV structure is examined through performing modal and fatigue analysis.

\section{Design of UAV structure}

A unified body model of UAV is created using SOLIDWORKS software which is shown in Figure 1. There is a significant opening for installing the hardware, and four spaces are accommodated for the four propellers at the edges of each arms. Presently, design space and non-design space are distinguished from the fundamental geometry for topological improvement. All the propeller openings, base plate, and edge of the base plate are chosen as non-design space. The rest of the configuration space has coincided with multi-zone work.

\subsection{Selection of 3D printing materials}

The present study envisaged to consider the AM technology and identification of high strength polymer to withstand the thrust load experienced by the UAV 


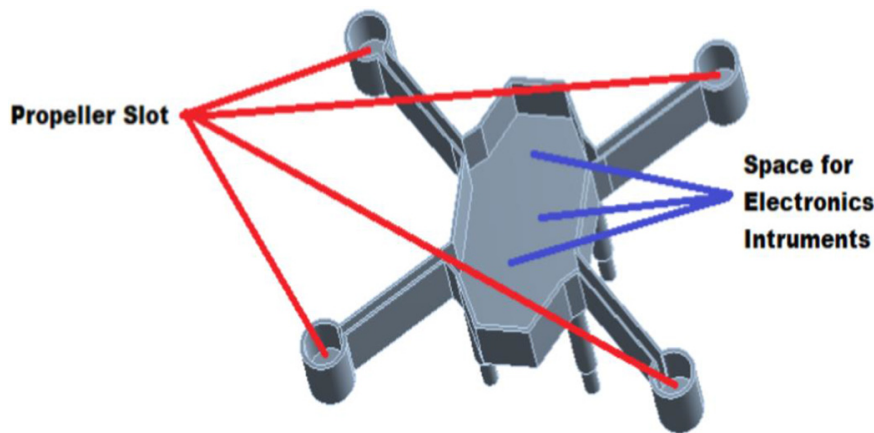

Table 1. Mechanical properties of ABS, POLYAMIDE \& PLA [33].

\begin{tabular}{llll}
\hline Property & ABS & PLA & Polyamide \\
\hline Density $\left(\mathrm{kg} \mathrm{m}^{-3}\right)$ & 1040 & 1290 & 1140 \\
Young modulus (MPa) & 2390 & 3500 & 1480 \\
Poisson ratio & 0.39 & 0.35 & 0.41 \\
Tensile yield strength (MPa) & 41.40 & 38 & 57.10 \\
Tensile ultimate strength (MPa) & 44.30 & 47.20 & 64.70 \\
\hline
\end{tabular}

Fig. 1. Design of UAV structure.
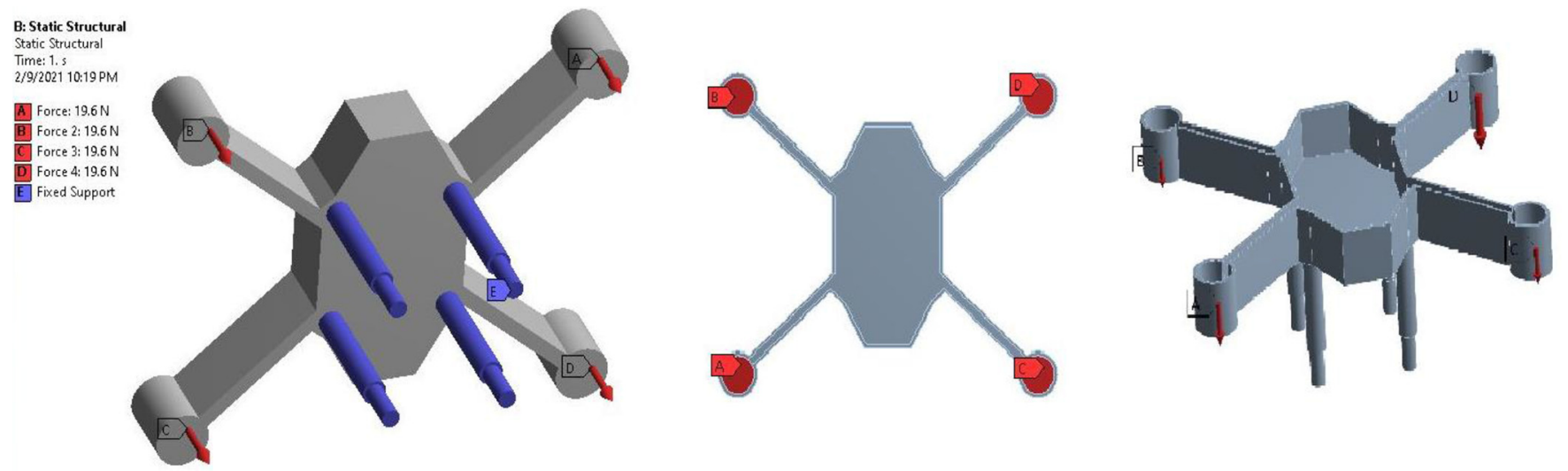

Fig. 2. Boundary conditions of UAV structure.

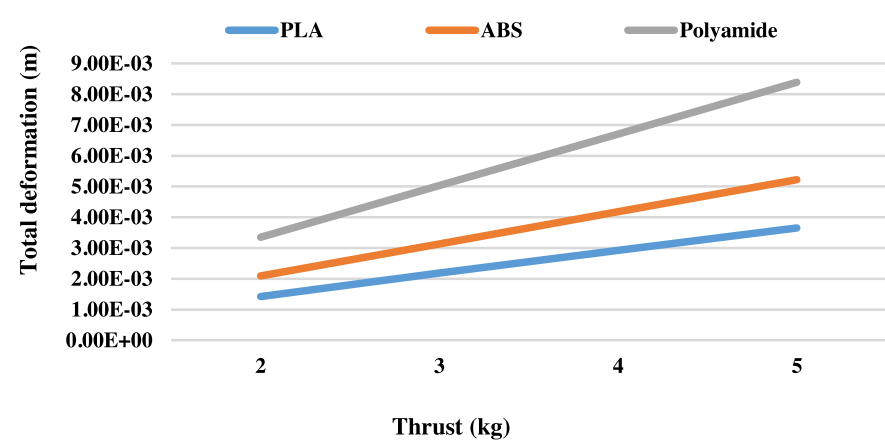

Fig. 3. Comparison of total deformation of UAV structure.

structure. Hence, it is essential to identify the functional materials which exhibit minimum structural behavior under the given loading conditions. In this study, three distinct polymer materials, such as Acrylonitrile Butadiene Styrene (ABS), Poly Lactic Acid (PLA), and Polyamide/ Nylon, are considered, and their mechanical properties are given in Table 1.

\subsection{Finite element analysis}

The static structural analysis is performed on the designed UAV structure using ANSYS software to determine total deformation and von-mises stress. The load description of the UAV structure is shown in Figure 2 and also mentioned below.

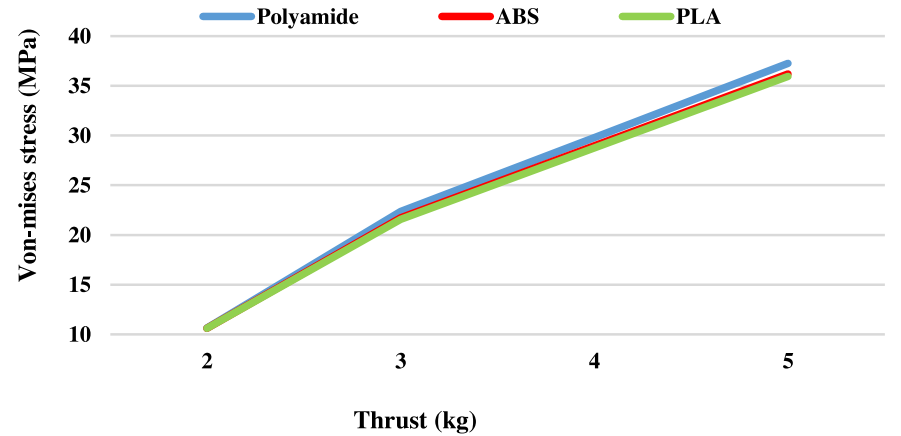

Fig. 4. Comparison of von Mises stress of UAV structure.

- The centerpiece of the lowermost plate is fixed.

- Arms appended with the base plate is fixed.

- Thrust of $5 \mathrm{~kg}$ is applied at each propeller.

Figure 3 shows the comparison of the total deformation of UAV structure for three polymer materials. It reveals that maximum total deformation occurred for polyamide whereas PLA achieved less total deformation with the value of $3.95 \mathrm{~mm}$ at $5 \mathrm{~kg}$ thrust.

Figure 4 shows the comparison of von-mises stress of UAV structure for three polymer materials. From static structural analysis, it is concluded that PLA exhibits better results compared to ABS and polyamide. Hence in this study, PLA is chosen as UAV structure material. 

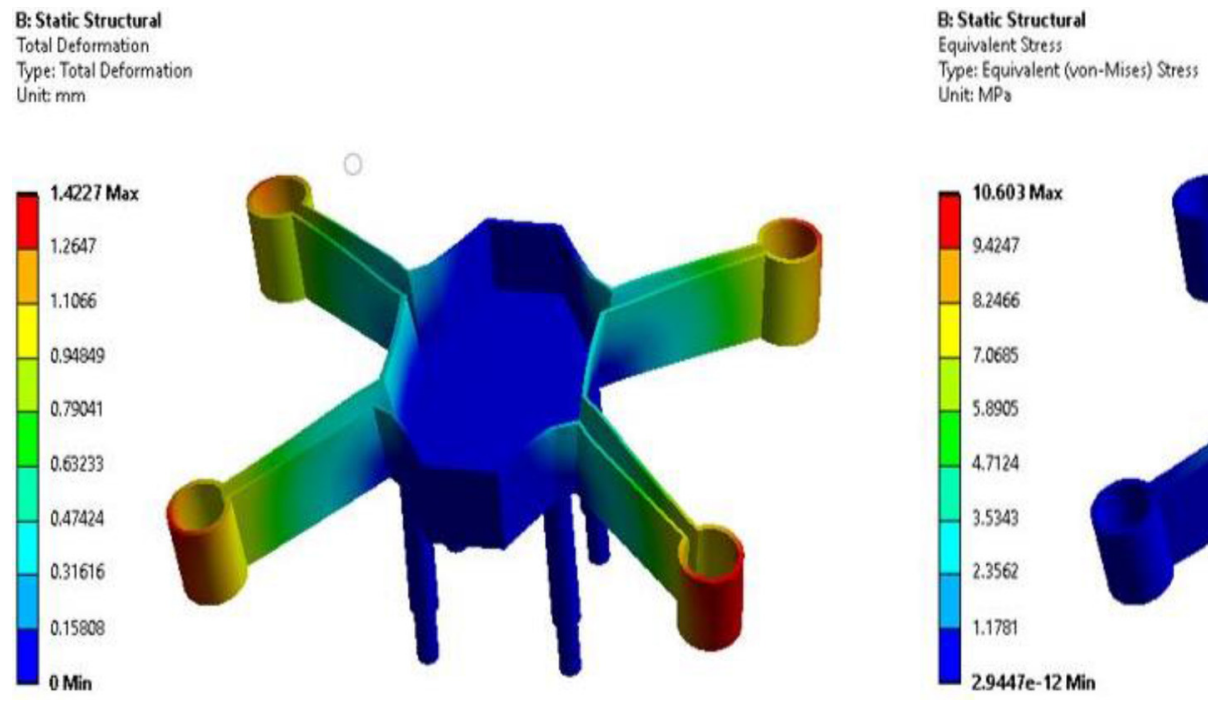

Fig. 5. Contour plot of total deformation and von Mises stress for PLA.

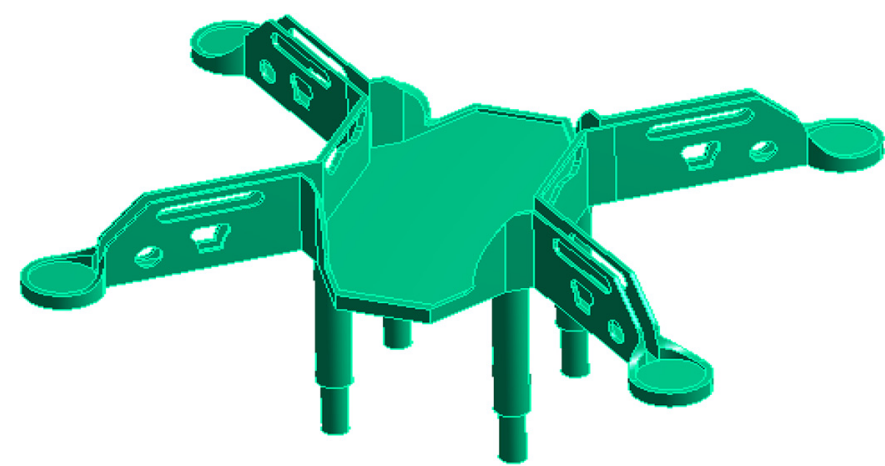

Fig. 6. Topologically optimized body.

Figure 5 shows contour plots of total deformation and von Mises stress of a UAV structure made of PLA. It can be seen that maximum deformation of $1.42 \mathrm{~mm}$ is obtained at the propeller casings, and maximum stress of $10.6 \mathrm{MPa}$ is obtained at the structure's base.

\section{Topology optimization}

The process of optimizing topology is a lengthy process requiring several rounds for optimal distribution. Topological optimization is achieved by reducing static compliance as a fitness function and reducing weight as a condition. To limit the boundaries, the mechanical behavior of the substance is utilized. The aim of topology optimization is usually to maximize rigidity and reduce its weight. Any construction optimization can help to reduce mass and contribute to a greater payload thrust. It is carried out by utilizing the Solid Isotropic Material with Penalization (SIMP) approach in ANSYS to optimize the stress-based topology. In general, there are three sorts of goal functions: weight reduction, volume reduction, and compliance minimization. The most commonly utilized approach is to reduce compliance, which is a synonym for maximizing stiffness. The reduction in compliance and mass is considered an objective function in this specific model.

The construction of the optimization problem is as follows:

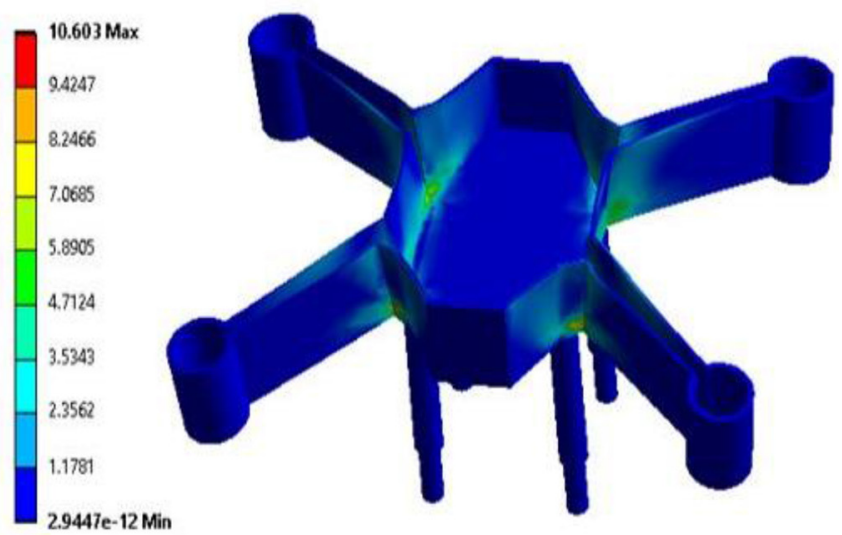

The optimization is performed by considering a union exactness of $0.1 \%$ and a safety factor of 3 . The upgraded model is accomplished after 21 iterations, as shown in Figure 6. After the improvement of the structure, the mass of the structure is reduced to $342.56 \mathrm{~g}$ from $387.65 \mathrm{~g}$.

\subsection{Confirmation study}

In order to validate the structural behavior of the optimized UAV structure static analysis is performed by considering similar boundary constraints, as shown in Figure 7.

\section{Results and discussion}

The static structural investigation has explored for the optimized UAV structure at different thrust conditions. The results show that similar structural behavior is displayed as in the preliminary design of the UAV structure. Figure 8 shows the comparison of the total deformation of UAV structure before and after optimization.

It is observed that the total deformation is expanding progressively as payload increases for both the conditions (before and after optimization). PLA experienced less 


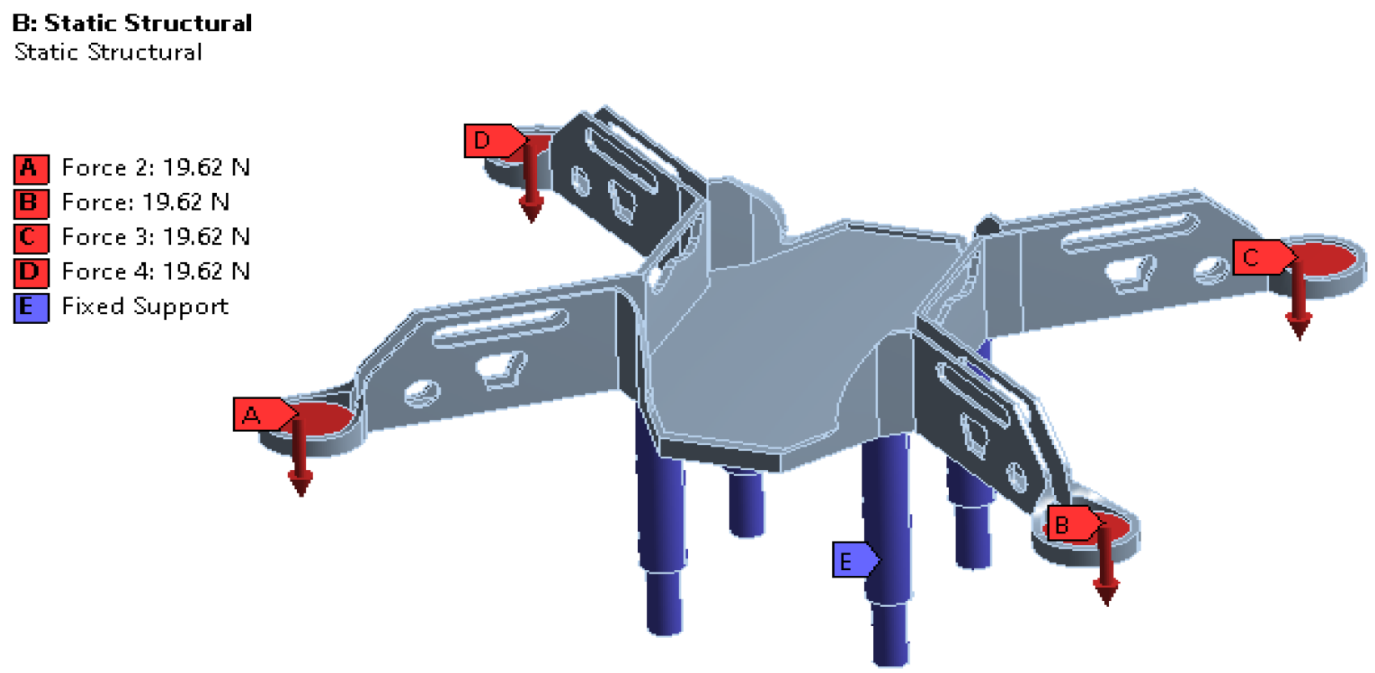

Fig. 7. Boundary conditions of optimized UAV structure.

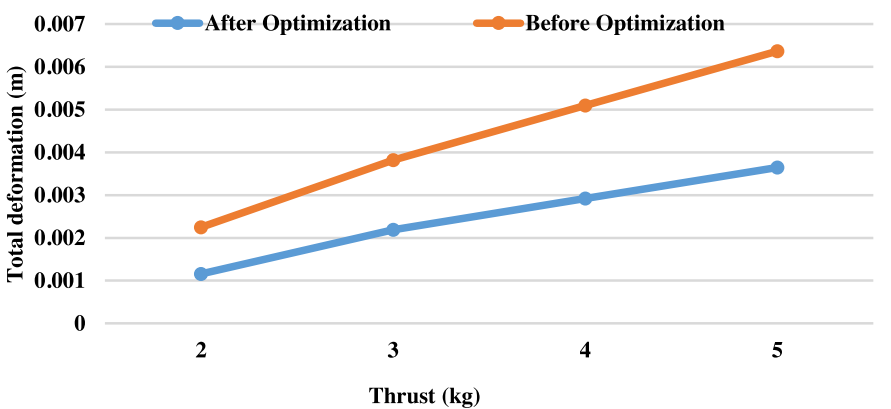

Fig. 8. Comparison on total deformation of UAV structure.

deformation ranging from $0.0011 \mathrm{~m}$ to $0.0038 \mathrm{~m}$ at four different payload conditions after topological optimization. The total deformation of PLA is minimized by $26 \%$ approximately with topological optimization.

The von Mises stress under different thrust conditions before and after optimization is examined for the PLA material. Besides, topological optimization implies an appreciable reduction in von Mises stress ranging from 10.25 MPa to 25.62 MPa as depicted in Figure 9.

Figure 10 demonstrates that the optimized UAV frame attained a distortion of $1.28 \mathrm{~mm}$ and von Mises stress of 11.49 MPa which confirms that the UAV structure can withstand the thrust load of $5 \mathrm{~kg}$ experienced by the UAV.

\section{Fatigue and vibrational characteristics of UAV structure}

In order to understand the structural rigidity of unified body of the optimized UAV structure under cyclic loads and also to examine the natural frequencies to avoid the mode of operation at those frequencies, FEA is performed.

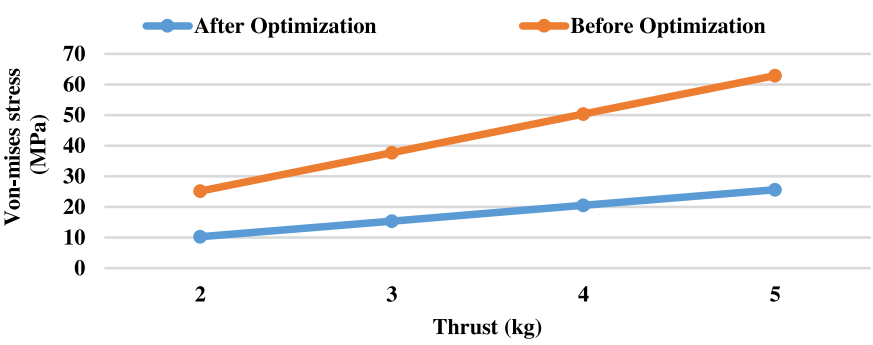

Fig. 9. Comparison on von Mises stress of UAV structure.

\subsection{Fatigue analysis}

Fatigue failure is one of the common causes of collapse of the engineering structure and especially UAV structure undergoes cyclic loads due to the rotation of propellers that will be transmitted to the central UAV frame. Fatigue analysis identifies the maximum number of cycles the UAV structure undergoes prior to its failure. As illustrated in Figure 11, total fatigue life is derived from the FEA simulation by applying data of attributes of the material given in Table 1 and the $\mathrm{S}-\mathrm{N}$ curve is constructed which is shown in Figure 11.

After performing fatigue analysis using ANSYS platform, the results predicted that, the UAV structure can withstand 1.5e5 cycles as depicted in Figure 12 under the cyclic loading experienced by the propellers.

\subsection{Vibrational analysis}

Modal analysis refers to evaluating vibrational properties such as natural frequencies and mode shapes of a mechanical structure. Therefore, modal analysis is performed for unified UAV body structure to identify the natural frequencies. Figure 13 shows the first five natural frequencies of the optimized structure which is far away from the operating frequency of $2.4 \mathrm{GHz}$. These natural frequencies are much larger than the working frequency of 


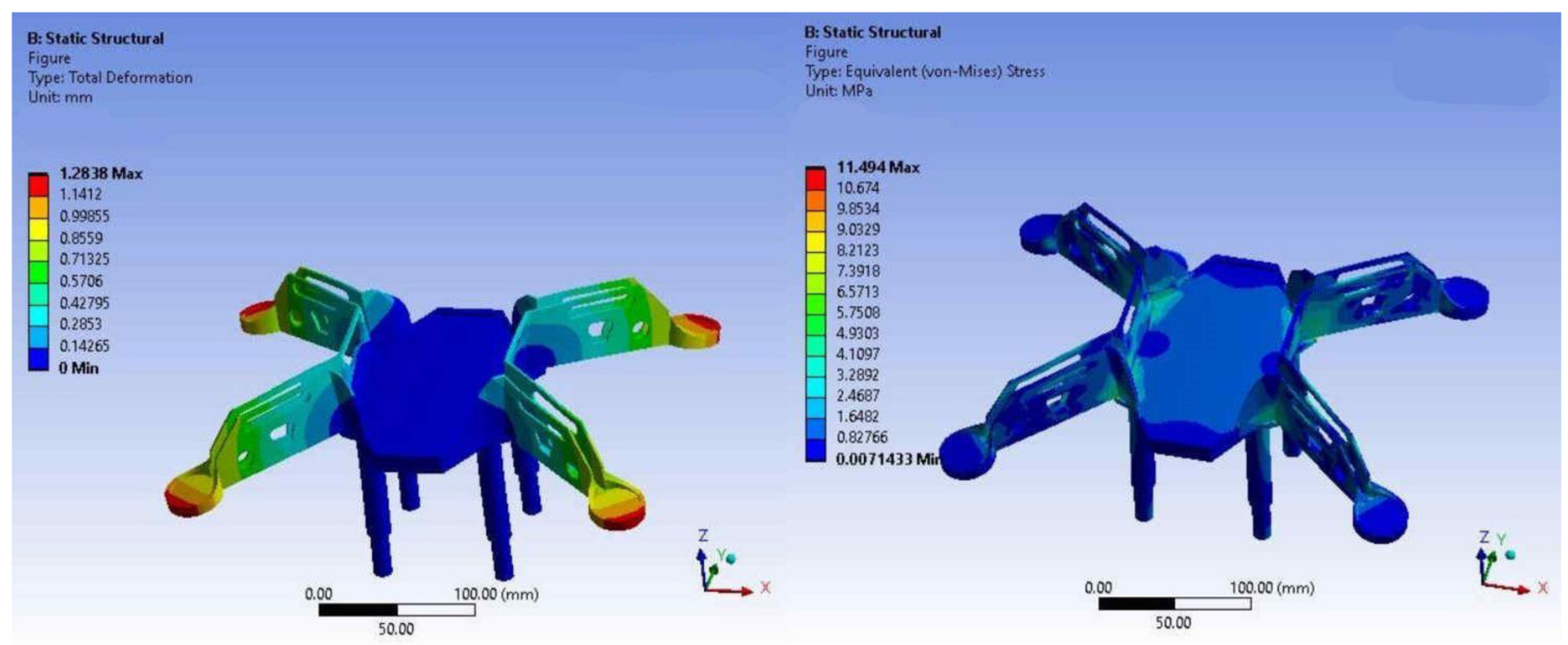

Fig. 10. Deformation and von Mises stress of optimized structure.

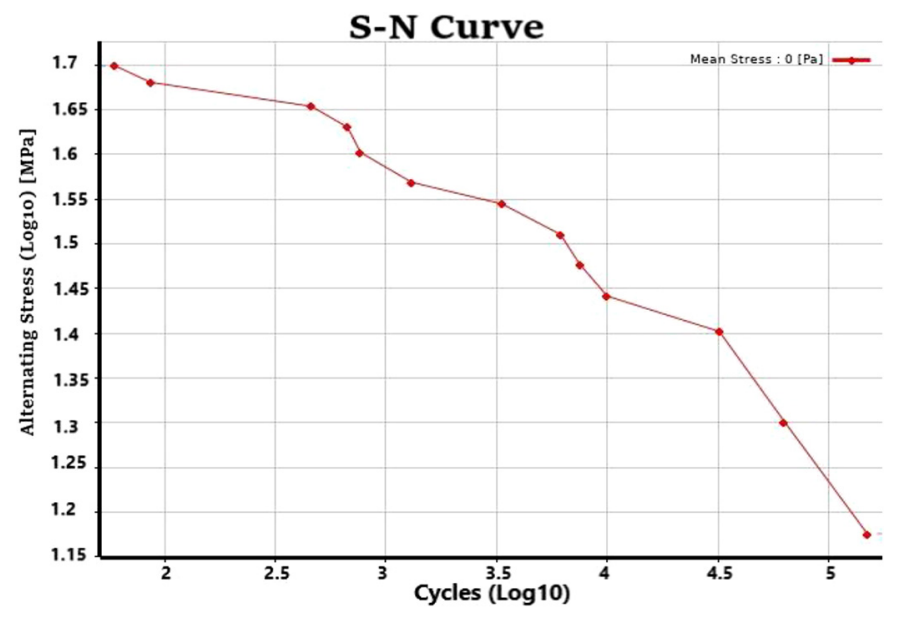

Fig. 11. S-N curve of PLA.

the rotating blades (may be less than $2300 \mathrm{RPM}=38 \mathrm{~Hz}$ ). Figures 14-16 illustrate first three modes and their natural frequencies such as the first mode experience pitching, second and third mode has achieved yawing.

\section{Conclusion}

The present work exploited optimization and simulation studies on Quadcopter structure that undergoes static and cyclic loads during its maneuvering. The comparative evaluation of well-established 3D printing materials such as PLA, ABS and polyamide confirms that, PLA out formed than other material with respect to less deformation and minimal stress occurrence under the $5 \mathrm{~kg}$ thrust load. The topological optimization studies yielded a $45 \mathrm{~g}$ reduction in mass in comparisons to initial weight of UAV structure. The unified optimized UAV structure can

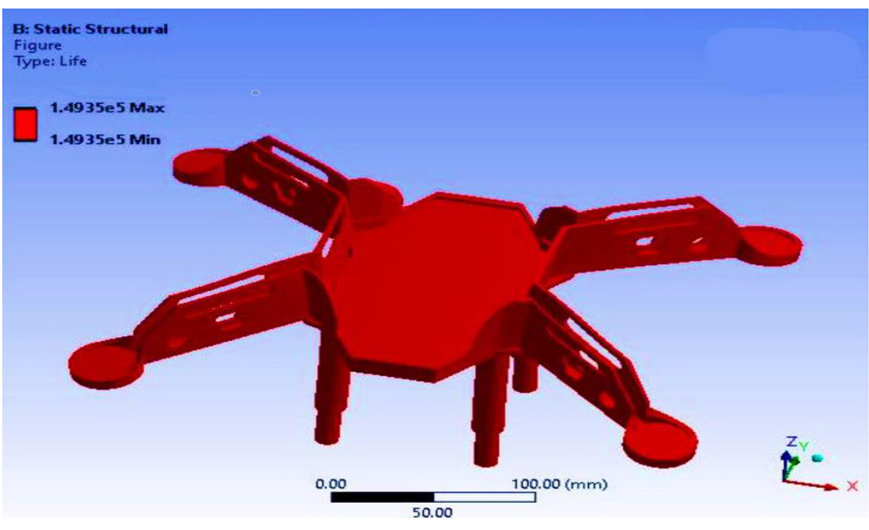

Fig. 12. Fatigue analysis: Maximum number of cycles.

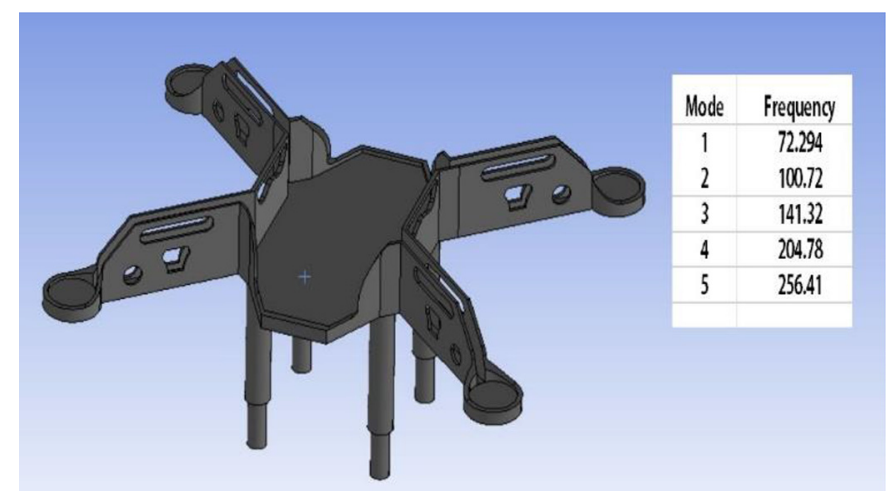

Fig. 13. Natural frequencies.

withstand the $1.5 \mathrm{e} 5$ cyclic loads and its first three natural frequencies $(72,100$ and $141 \mathrm{~Hz})$ is far away from the operating frequency of $2.4 \mathrm{GHz}$. Hence, the optimal UAV structure can be $3 \mathrm{D}$ printed and utilized for navigation in real time. Due to the weight reduction and monocoque 


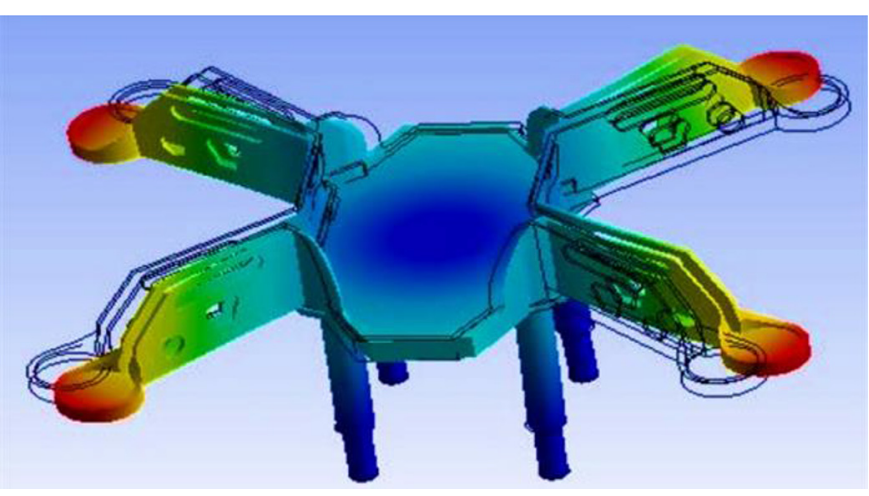

Fig. 14. First mode shape and its corresponding frequency at $72.294 \mathrm{~Hz}$.

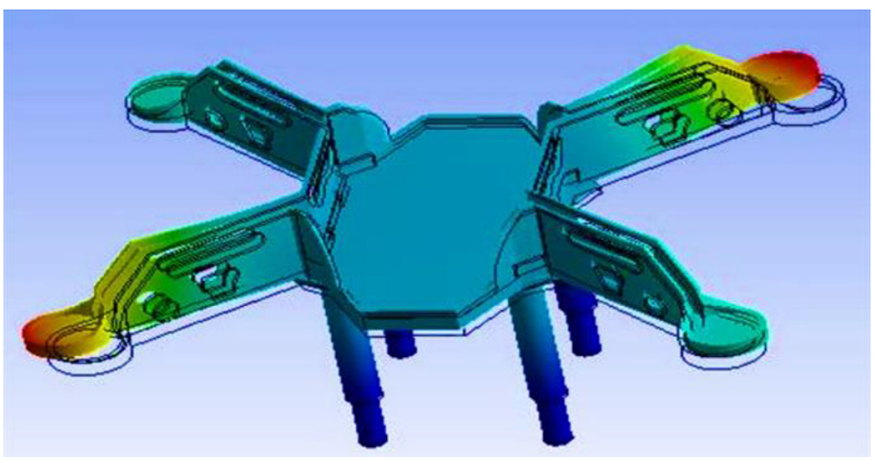

Fig. 15. Second mode shape and its corresponding frequency at $100.72 \mathrm{~Hz}$

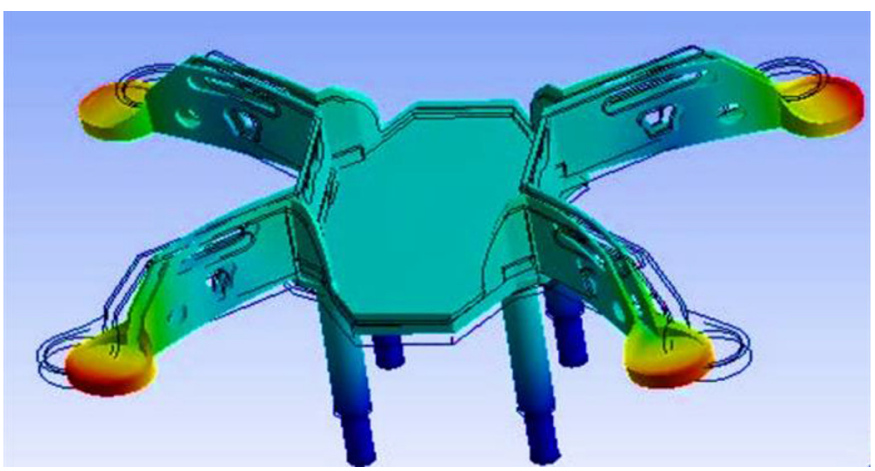

Fig. 16. Third mode shape and its corresponding frequency at $141.32 \mathrm{~Hz}$

UAV structure, it can increase the flight time and reduce the assembly effort by avoiding the bolt and nut connections. In future, the optimized structure will be $3 \mathrm{D}$ printed and real time flight trails will be conducted to evaluate the aerodynamic performance characterizes of the developed optimized UAV structure.

\section{References}

1. H. Shakhatreh, A.H. Sawalmeh, A. Al-Fuqaha, Z. Dou, E. Almaita, I. Khalil, M. Guizani, Unmanned aerial vehicles (UAVs): A survey on civil applications and key research challenges, IEEE Access 7, 48572-48634 (2019)

2. P. Radoglou-Grammatikis, P. Sarigiannidis, T. Lagkas, I. Moscholios, A compilation of UAV applications for precision agriculture, Comput. Netw. 172, 107148 (2020)

3. S. Park, Y. Choi, Applications of unmanned aerial vehicles in mining from exploration to reclamation: a review, Minerals 10, $663(2020)$

4. A. Valsan, B. Parvathy, R.S. Unnikrishnan, P.K. Reddy, A. Vivek, Unmanned aerial vehicle for search and rescue mission, in 2020 4th International Conference on Trends in Electronics and Informatics (ICOEI) (48184). IEEE (2020, June), pp. 684-687

5. A. Sigala, B. Langhals, Applications of Unmanned Aerial Systems (UAS): a Delphi Study projecting future UAS missions and relevant challenges, Drones 4, 8 (2021)

6. B. Esakki, S. Mathiyazhagan, M. Moses, K.J. Rao, S. Ganesan, Development of 3D-printed floating Quadrotor for collection of algae in remote water bodies, Comput. Electr. Agric. 164, 104891 (2019)

7. E. Balasubramanian, N.V.S.S. Sagar, U. Chandrasekhar, S. Salunkhe, Development of light weight multi-rotor UAV structures through synergistic application of design analysis and fused deposition modeling, Int. J. Mater. Product Technol. 59, 229-238 (2019)

8. H. Klippstein, A. Diaz De Cerio Sanchez, H. Hassanin, Y. Zweiri, L. Seneviratne, Fused deposition modeling for unmanned aerial vehicles (UAVs): a review, Adv. Eng. Mater. 20, 1700552 (2018)

9. A.W. Gebisa, H.G. Lemu, A case study on topology optimized design for additive manufacturing, in Materials Science and Engineering Conference Series (2017, December), Vol. 276, No. 1, p. 012026

10. G.I. Rozvany, A critical review of established methods of structural topology optimization, Struct. Multidiscipl. Optim. 37, 217-237 (2009)

11. O. Sigmund, K. Maute, Topology optimization approaches, Struct. Multidiscipl. Optim. 48, 1031-1055 (2013)

12. J. Liu, A.T. Gaynor, S. Chen, Z. Kang, K. Suresh, A. Takezawa, L. Cheng, Current and future trends in topology optimization for additive manufacturing, Struct. Multidiscipl. Optim. 57, 2457-2483 (2018)

13. M.P. Bendsøe, O. Sigmund, Material interpolation schemes in topology optimization, Arch. Appl. Mech. 69, 635-654 (1999)

14. M.Y. Wang, X. Wang, D. Guo, A level set method for structural topology optimization, Comp. Methods Appl. Mech. Eng. 192, 227-246 (2003)

15. G. Allaire, F. Jouve, A.M. Toader, Structural optimization using sensitivity analysis and a level-set method, J. Comput. Phys. 194, 363-393 (2004)

16. J. Liu, Y. Ma, A survey of manufacturing oriented topology optimization methods, Adv. Eng. Softw. 100, 161-175 (2016)

17. B.S. Lazarov, F. Wang, O. Sigmund, Length scale and manufacturability in density-based topology optimization, Arch. Appl. Mech. 86, 189-218 (2016)

18. G. Allaire, F. Jouve, G. Michailidis, Thickness control in structural optimization via a level set method, Struct. Multidiscip. Optim. 53, 1349-1382 (2016) 
19. J.K. Guest, J.H. Prévost, T. Belytschko, Achieving minimum length scale in topology optimization using nodal design variables and projection functions, Int. J. Numer. Methods Eng. 61, 238-254 (2004)

20. J.K. Guest, Imposing maximum length scale in topology optimization, Struct. Multidiscip. Optim. 37, 463-473 (2009)

21. X. Guo, W. Zhang, W. Zhong, Explicit feature control in structural topology optimization via level set method, Computer Methods Appl. Mech. Eng. 272, 354-378 (2014)

22. Q. Xia, T. Shi, M.Y. Wang, S. Liu, A level set based method for the optimization of cast part, Struct. Multidiscip. Optim. 41, 735-747 (2010)

23. J. Liu, Y.S. Ma, 3D level-set topology optimization: a machining feature-based approach, Struct. Multidiscip. Optim. 52, 563-582 (2015)

24. G.D. Goh, S. Agarwala, G.L. Goh, V. Dikshit, S.L. Sing, W.Y. Yeong, Additive manufacturing in unmanned aerial vehicles (UAVs): challenges and potential, Aerospace Sci. Technol. 63, 140-151 (2017)

25. S.K. Moon, Y.E. Tan, J. Hwang, Y.J. Yoon, Application of 3D printing technology for designing light-weight unmanned aerial vehicle wing structures, Int. J. Precis. Eng. Manufactur. Green Technol. 1, 223-228 (2014)

26. N.V.S.S. Sagar, K.S. Vepa, Experimental investigations for improving the strength of parts manufactured using FDM process, in Innovative Design, Analysis and Development
Practices in Aerospace and Automotive Engineering (I-DAD 2018). Springer, Singapore (2019) pp. 307-313

27. N.A. Ahmed, J.R. Page, Manufacture of an unmanned aerial vehicle (UAV) for advanced project design using 3D printing technology, in Applied Mechanics and Materials, Trans Tech Publications 397, 970-980 (2013).

28. S.H. Ahn, M. Montero, D. Odell, S. Roundy, P.K. Wright, Anisotropic material properties of fused deposition modeling ABS, Rapid Prototyp. J. 8, 248-257 (2002)

29. K. Chockalingam, N. Jawahar, J. Praveen, Enhancement of anisotropic strength of fused deposited ABS parts by genetic algorithm, Mater. Manufactur. Processes 31, 2001-2010 (2016)

30. L.J. Yang, The micro-air-vehicle Golden Snitch and its figure-of-8 flapping, J. Appl. Sci. Eng. 15, 197-212 (2012)

31. F.Y. Hsiao, L.J. Yang, S.H. Lin, C.L. Chen, J.F. Shen, Autopilots for ultra light weight robotic birds- Automatic altitude control and system integration of a sub- $10 \mathrm{~g}$ weight flapping wing micro air vehicle, IEEE Control Syst. Mag. 32, $35-48(2012)$

32. L.J. Yang, C.Y. Kao, C.K. Huang, Development of flapping ornithopters by precision injection molding, Appl. Mech. Mater. 163, 125-132 (2012)

33. MATWEB. (n.d.). Retrieved from Material Property Data: http://www.matweb.com/index.aspx

Cite this article as: Ashraf Mahmud Rayed, Balasubramanian Esakki, Arunkumar Ponnambalam, Sajal Chandra Banik, Karim Aly, Optimization of UAV structure and evaluation of vibrational and fatigue characteristics through simulation studies, Int. J. Simul. Multidisci. Des. Optim. 12, 17 (2021) 